\section{Pacific Northwest}

National Laboratory

Operated by Battelle for the

U.S. Department of Energy

\title{
Progress Report on Frequency - Modulated Differential Absorption Lidar
}

\author{
BD Cannon MS Taubman \\ WW Harper RM Williams \\ TL Myers JF Schultz
}

December 2001

Prepared for the U.S. Department of Energy under Contract DE-AC06-76RL01830 


\title{
DISCLAIMER
}

This report was prepared as an account of work sponsored by an agency of the United States Government. Neither the United States Government nor any agency thereof, nor Battelle Memorial Institute, nor any of their employees, makes any warranty, express or implied, or assumes any legal liability or responsibility for the accuracy, completeness, or usefulness of any information, apparatus, product, or process disclosed, or represents that its use would not infringe privately owned rights. Reference herein to any specific commercial product, process, or service by trade name, trademark, manufacturer, or otherwise does not necessarily constitute or imply its endorsement, recommendation, or favoring by the United States Government or any agency thereof, or Battelle Memorial Institute. The views and opinions of authors expressed herein do not necessarily state or reflect those of the United States Government or any agency thereof.

\author{
PACIFIC NORTHWEST NATIONAL LABORATORY \\ operated by \\ BATTELLE \\ for the \\ UNITED STATES DEPARTMENT OF ENERGY \\ under Contract DE-AC06-76RL01830
}

Printed in the United States of America

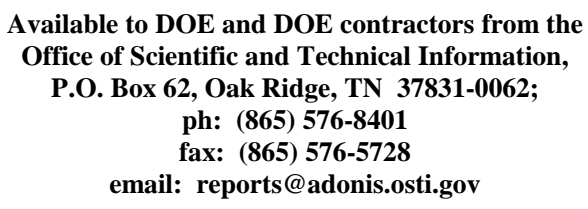

Available to the public from the National Technical Information Service, U.S. Department of Commerce, 5285 Port Royal Rd., Springfield, VA 22161 ph: (800) 553-6847 fax: $(703) 605-6900$

email: orders@ntis.fedworld.gov

online ordering: http://www.ntis.gov/ordering.htm 


\section{Progress Report on Frequency - Modulated Differential Absorption Lidar}
B. D. Cannon
M. S. Taubman
W. W. Harper
R. M. Williams
T. L. Myers
J. F. Schultz

December 2001

Prepared for

the U.S. Department of Energy

under Contract DE-AC06-76RLO 1830

Pacific Northwest National Laboratory

Richland, Washington 99352 



\section{Summary}

Modeling done at Pacific Northwest National Laboratory (PNNL) in FY2000 predicted improved sensitivity for remote chemical detection by differential absorption lidar (DIAL) if frequency-modulated (FM) lasers were used. This improved sensitivity results from improved averaging of speckle noise. The recently developed quantum cascade (QC) lasers offer the first practical method for implementing this approach in the molecular fingerprint region of the infrared. To validate this model prediction, a simple laboratory bench FM-DIAL system was designed, assembled, tested, and laboratory-scale experiments were carried out during FY2001. Preliminary results of the FM DIAL experiments confirm the speckle averaging advantages predicted by the models. In addition, experiments were performed to explore the use of hybrid QC - $\mathrm{CO}_{2}$ lasers for achieving sufficient frequency-modulated laser power to enable field experiments at longer ranges (up to one kilometer or so). This approach will allow model validation at realistic ranges much sooner than would be possible if one had to first develop master oscillator - power amplifier systems utilizing only QC devices. Amplification of a QC laser with a $\mathrm{CO}_{2}$ laser was observed in the first hybrid laser experiments, but the low gain and narrow linewidth of the $\mathrm{CO}_{2}$ laser available for these experiments prevented production of a high-power FM laser beam. 



\section{Contents}

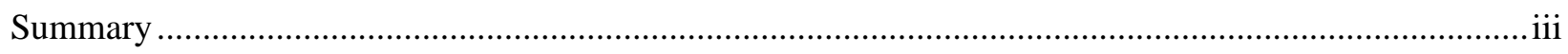

1.0 Introduction, Objectives, and Summary of Work Performed to Date .............................................. 1

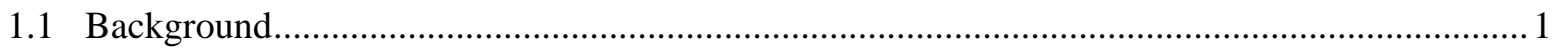

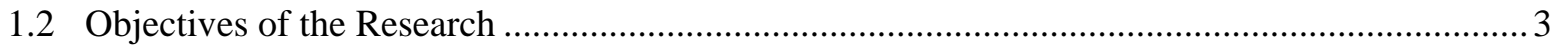

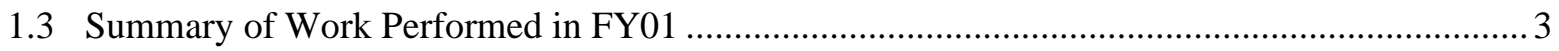

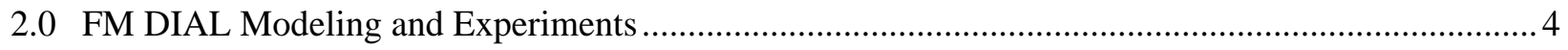

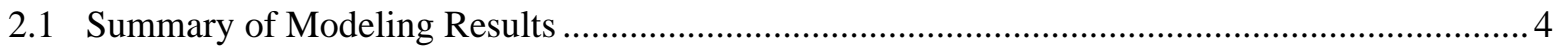

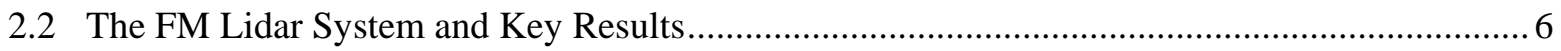

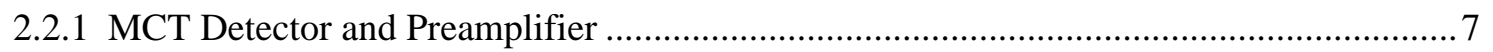

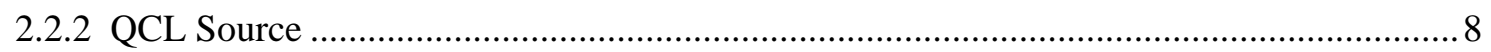

2.3 Initial Observations of Laser Speckle …........................................................................ 9

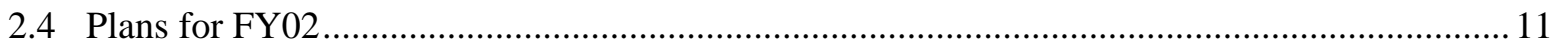

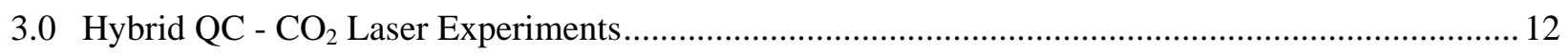

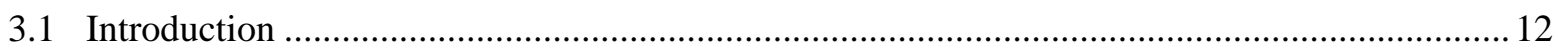

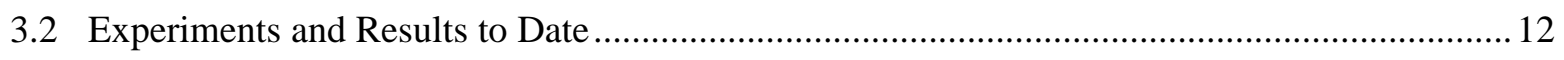

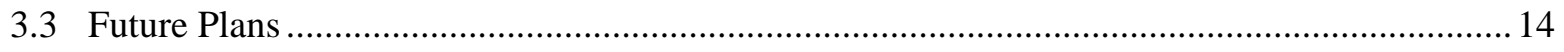

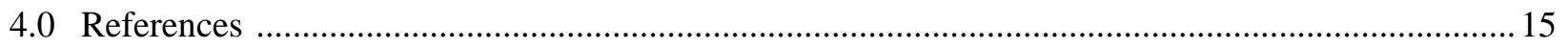




\section{Figures}

1. Derivatives of Chemical Absorption Spectra are More Distinctive than the Absorption Spectra Themselves

2. Derivatives of Even Relatively Featureless Spectra are Much More Distinctive.

3. Numerical Model Block Diagram for FM-LIDAR

4. The Source is a Quantum Cascade Laser Operating at a Wavelength of $8.2 \mu \mathrm{m}$ and Capable of Delivering up to $30 \mathrm{~mW}$ of Continuous-Wave Power in a Narrow Optical Bandwidth.....

5. Each Trace was Converted to $\mathrm{V}_{\mathrm{rms}} /(\mathrm{Hz})^{1 / 2}$, and Divided by $5200 \Omega$ to get an Input Referred Current Noise

6. Phase Shift for the Residual Amplitude Modulation and Frequency Modulation

7. Diagram for Studying Laser Speckle .......

8. Single Foam Target Speckle Pattern Taken with an IR Bolometer Camera

9. Frequency-Dependent Cycling of Laser Speckle.

10. Amplified Power Output vs. QC Power Input for a Single-Pass $\mathrm{CO}_{2} \mathrm{Amplifier}$

11. Experimental Set-Up for Injection Locking

12. FTIR Spectrum for the Superposed Output of the QC and the $\mathrm{CO}_{2}$ Laser. 


\subsection{Introduction, Objectives, and Summary of Work Performed to Date}

\subsection{Background}

In an idealized differential absorption lidar (DIAL) measurement, a molecular absorption is measured using light at a pair of closely spaced optical frequencies that is transmitted through the atmosphere, scattered from the ground or aerosols, and then transmitted back through the atmosphere to a detector near the transmitter. One of these frequencies is on a molecular absorption, while the other is detuned from the resonance, and because the frequencies are so close together, the backscattering efficiencies of both optical frequencies are essentially the same. Thus, the ratio of detected powers at these two frequencies, after correction for the differences in transmitted power, is a measurement of the molecular absorption along the path of the light. In real applications, there are usually non-zero "background" absorptions that are different at every wavelength due to natural constituents or pollutants in the atmosphere, and spectral dependence in surface reflectivity. In addition, more than one chemical is usually present in the chemical effluent plume of interest, and in many cases the spectra of the different chemicals overlap at key wavelengths. These complications can be addressed, at least in principle, by interrogating the scene with multiple laser wavelengths of light and fitting the background effects to derive corrections to the detection wavelengths. The effectiveness of such techniques depends on the signal-to-noise ratio (SNR) in the data and the extent to which the spectral features of all the chemicals that might be present in the scene are distinct from each other and the background at the laser wavelengths used in the DIAL measurement. However, any such analysis requires that the backscattering efficiencies for all the wavelengths be correlated. Any uncorrelated variations in the backscattering efficiencies are multiplicative noise in the measurements. Laser speckle causes such uncorrelated variations in the backscattering efficiencies, and speckle is often the largest noise source in DIAL. Speckle is the interference between light waves that all have the same frequency arriving at a surface with random phase relationships between all the waves, such as when a laser beam is scattered from a rough surface.

In FY00, PNNL started modeling the use of frequency modulation (FM) spectroscopy to enhance the sensitivity and selectivity of DIAL techniques (Sheen 2000). The models developed predicted FM techniques could significantly reduce speckle noise, which could allow the options of lower detection

limits and faster measurements. Faster measurements would reduce the effects of background variability, which is another important noise source. FM techniques have been used for sensitive absorption measurements in laboratories for many years because this inherently AC (alternating current) method is free from many of the low frequency sources of noise (drift, vibrations, position variations, $1 / f$ noise). FM spectroscopy is also a derivative technique that senses the slopes of spectral features. As shown in Figures 1 and 2, the derivatives of even relatively featureless chemical spectra tend to be much more distinctive than the spectra themselves, which increases selectivity in the presence of interferences. Recent PNNL spectral database measurements have shown that several key molecules involved in chemical and nuclear weapons production have sharp $Q$-branch transitions. In these branches, successive transitions tend to overlap and 


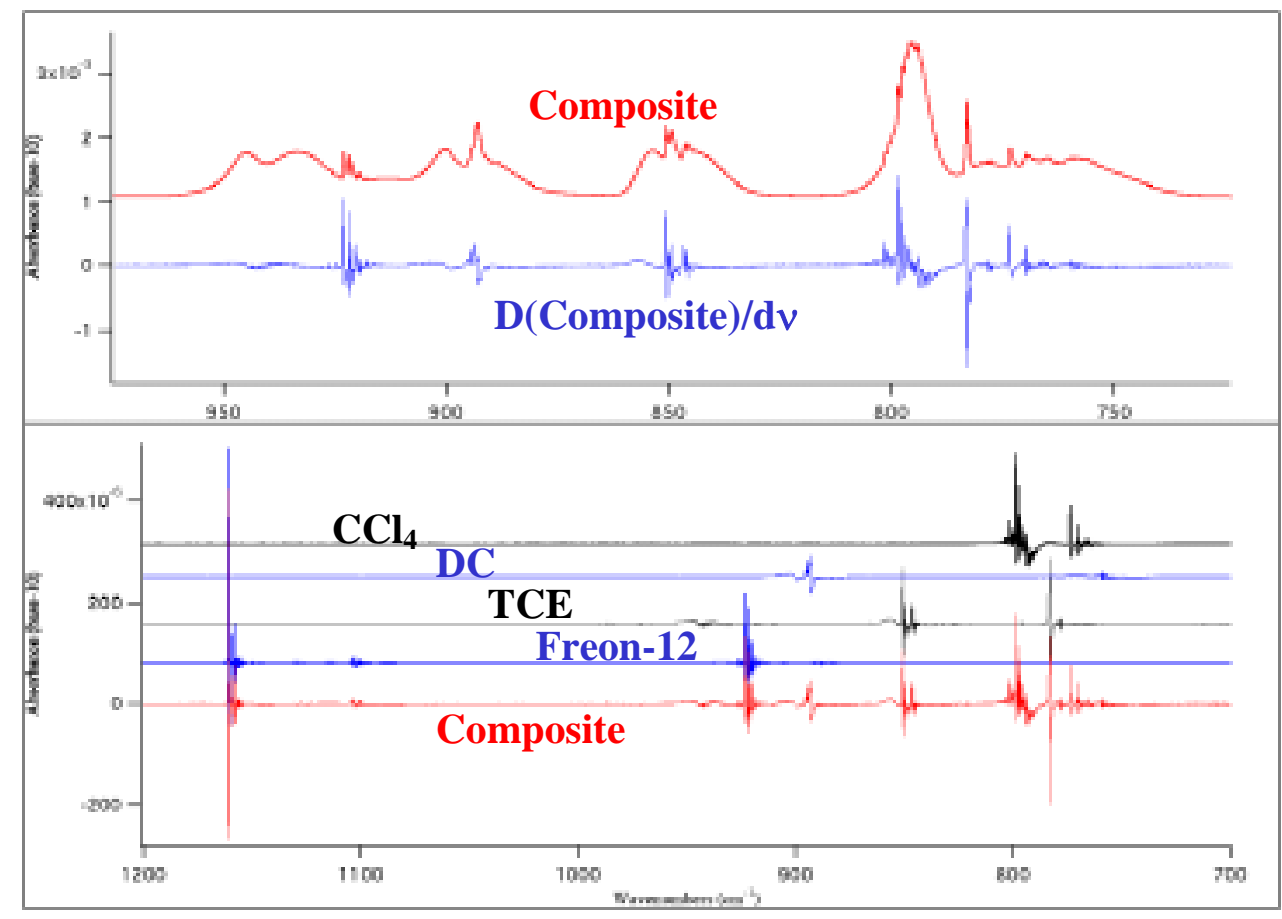

Figure 1. Derivatives of Chemical Absorption Spectra are More Distinctive than the Absorption Spectra Themselves

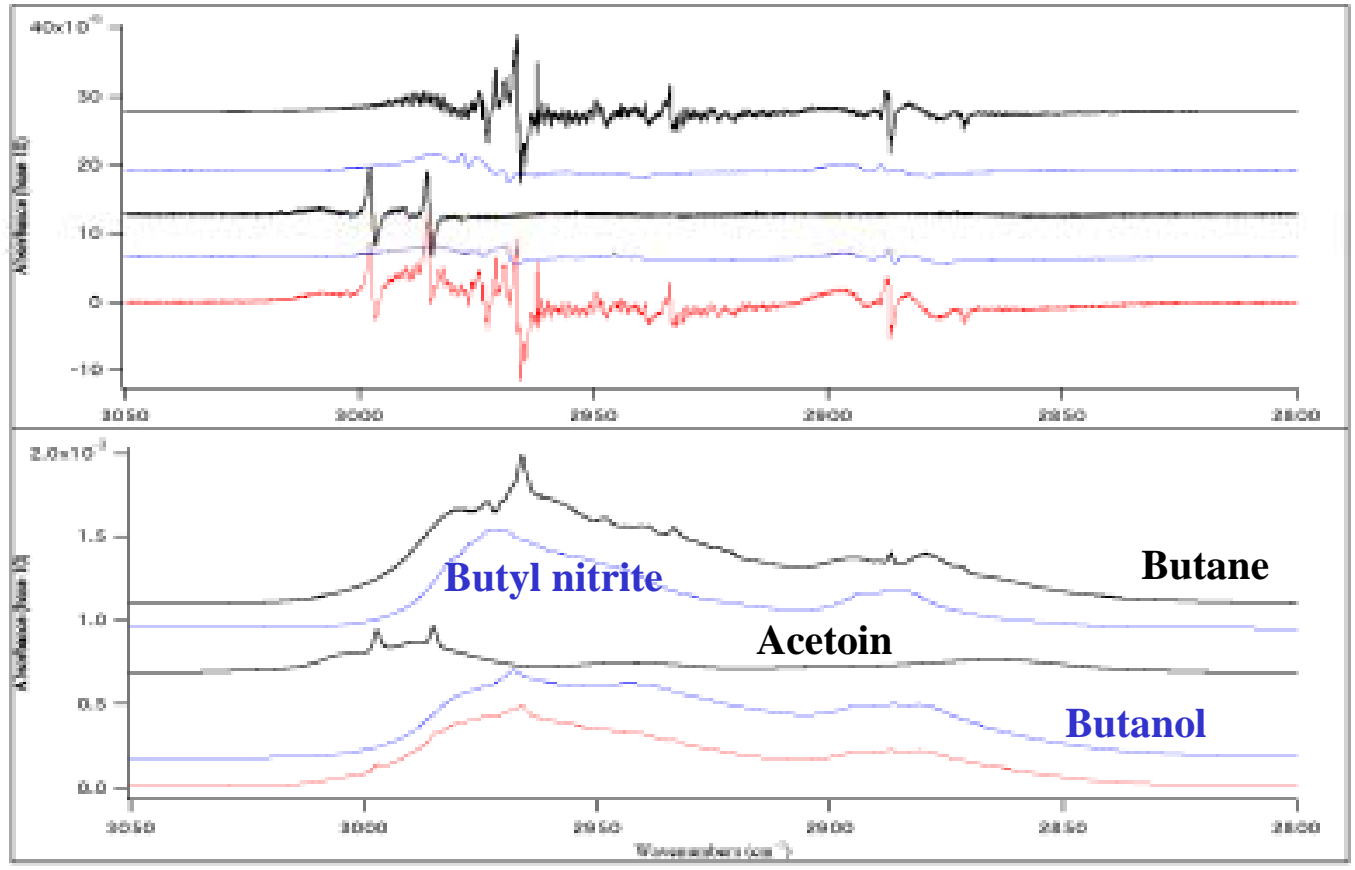

Figure 2. Derivatives of Even Relatively Featureless Spectra are Much More Distinctive 
increase the absorption intensity while the absorption width stays narrow, making a sharp and strong spectral feature. Because of the increased selectivity, the chemometrics and statistical analysis necessary to interpret the data may be greatly simplified.

\subsection{Objectives of the Research}

Based on recent numerical modeling work at PNNL (described below), FM spectroscopy techniques have the potential to either achieve sensitivities comparable to conventional DIAL in as little as one onehundredth of the target dwell time, or to achieve up to ten times the sensitivity in the same dwell time. In addition, chemical selectivity in the presence of background absorptions should be much greater because of the advantages of derivative spectroscopy inherent in FM techniques, thus reducing the incidence of false positives and false negatives. Quantum cascade (QC) lasers, with adequate stability, power, and tunability in the critical mid-wave infrared (MWIR - wavelengths lying between 3 and 14 microns) spectral region where atmospheric transmission windows and the most distinctive molecular spectra coincide, now make it possible to consider FM spectroscopy techniques for practical chemical sensing systems. The objectives of this work are:

1) To explore the phenomenology of FM DIAL in practical field environments.

2) To validate and quantify the advantages predicted by numerical modeling in progressively realistic operating environments.

3) To investigate fundamental engineering and component technology issues.

As this work is completed, the results will be progressively incorporated in PNNL's Infrared Systems Analysis in General Environments (IR-SAGE) code to quantify the benefits of FM DIAL systems, optimize designs for specific applications, and permit valid comparison to other techniques.

\subsection{Summary of Work Performed in FY01}

During FY01, a simple laboratory bench lidar was designed, the components and subsystems were carefully characterized, and laboratory-scale experiments were carried out to validate the improvements in speckle averaging predicted by the models. In addition, experiments were performed to explore the use of hybrid QC - $\mathrm{CO}_{2}$ lasers for achieving sufficient laser power to enable field experiments at longer ranges. Preliminary results of the FM DIAL experiments confirm the speckle averaging advantages predicted by the models. Amplification of a $\mathrm{QC}$ laser with a $\mathrm{CO}_{2}$ laser was observed in the first hybrid laser experiments, but the gain and linewidth of the $\mathrm{CO}_{2}$ laser available for these experiments was too low to provide effective injection locking. 


\subsection{FM DIAL Modeling and Experiments}

\subsection{Summary of Modeling Results}

A block diagram of our numerical model of FM-LIDAR is shown in Figure 3. At present, the modulation source is treated as a pure sine wave of known frequency $(f)$ that induces frequency modulation (FM) and amplitude modulation (AM) on the output of the transmitter laser. The value of $f$, the FM and AM amplitudes, and their phases relative to the modulation source are all user-adjustable parameters in the model. The physical model for the modulation predicts an amplitude modulation of the laser at $2 f$, with amplitude and phase are determined by the AM amplitude and phase at $1 f$, but no modulation at higher harmonics of $f$. The relationships among the FM and AM amplitudes and phases and their dependence on $f$ needs to be measured, especially for quantum cascade lasers (QCLs) as does the presence or absence of higher harmonics of the modulation frequency. The detector and preamplifier are currently modeled by specifying a size and quantum efficiency for the detector, a trans-impedance gain for the preamplifier, and including a number of white noise terms such as shot noise from thermal black body emission, shot noise from leakage current, and Johnson noise from the feedback resistor in the preamp. Other noise sources, such as op-amp current and voltage noise, $1 / f$ noise, and departures of the mercury-cadmium-telluride detector from the normal idealized model, as well as the details of the preamplifier design, were omitted until experiments could identify and characterize the important issues. The transmitter and receiver optics are simply modeled as having a user-specified size and transmission, though further refinements may need to be added in the future. The atmosphere is modeled as having turbulence that changes beam pointing and absorption that contains both sharp molecular features and broadband attenuation. Testing the effects of turbulence will require field tests at ranges of a kilometer or greater. Another aspect of long distance transmission through the atmosphere

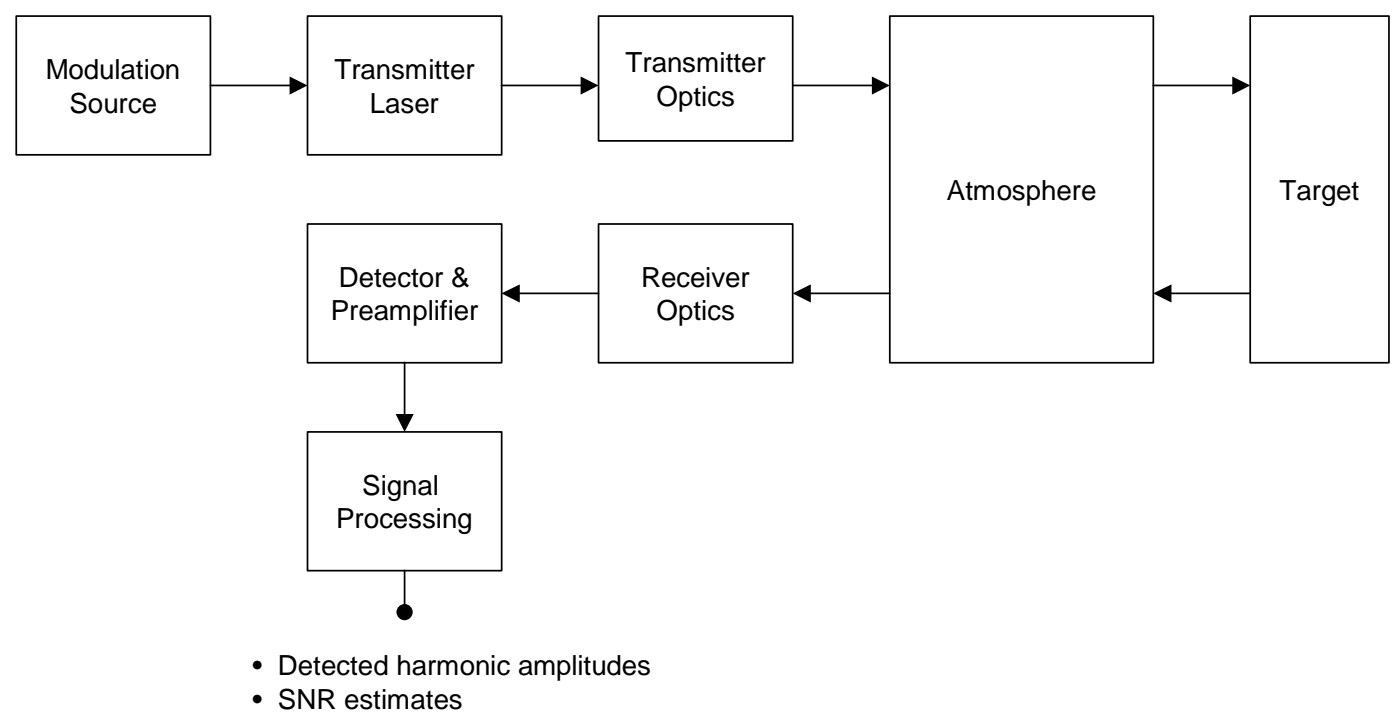

Figure 3. Numerical Model Block Diagram for FM-LIDAR 
that may need to be included in our model is loss of phase coherence of the FM beam. This point was raised by John Hall, who is a staff member at the Joint Institute for Laboratory Astrophysics and National Institute of Standards and Technology (NIST) in Boulder, Colorado, and a pioneer in using FM techniques with lasers.

The target module includes the possibilities for diffuse (Lambertian) scatter from a surface characterized by an albedo, a retro-reflector, and the bi-static case. For diffuse scatter, speckle is introduced into the model, and this is an important part of the SNR computation. Even with speckle present, a small enough area on the detector will have a uniform irradiance from the scattered light, but at a large enough distance away the irradiance on the detector could be completely different. The area over which the irradiance is correlated is called a speckle or speckle lobe. Similarly, by moving the transmitting laser, receiving optics, or changing the optical frequency a significant amount, the irradiance on a particular small area of the detector can change randomly and must be described statistically. The distribution of the irradiances in a single speckle is characterized by a mean and a standard deviation that is equal to the mean. Thus, a measurement of the power in a single speckle has a SNR of at most 1, independent of detector power and noise levels. Improving the SNR of the backscattered light measurement for a given optical frequency requires averaging the power in many speckles by having many speckles on the detector at one time and/or averaging many independent measurements. Independent measurements can be obtained by moving the optics between measurements assuming there are no correlations between the speckles of separate measurements. This has been thoroughly explored in work with DIAL using a $\mathrm{CO}_{2}$ laser where there are a large number of fixed optical frequencies. A key prediction from PNNL's model is that FM can increase the number of speckles included in the average by a moderate factor. The SNR due to speckle for measuring the backscattered light in a DIAL measurement a single FM modulated laser frequency is:

$$
S N R_{\text {speckle }}=\sqrt{N_{\text {spatial }} \cdot N_{\text {meas }} \cdot N_{v}}
$$

where $N_{\text {spatial }}$ is the number of speckles on the detector, $N_{\text {meas }}$ is the number of uncorrelated measurements, and $N_{v}$ is the factor by which the number of speckles on the detector is increased by modulating the frequency. The factors $N_{\text {spatial }}$ and $N_{\text {meas }}$ have been modeled and verified previously with $\mathrm{CO}_{2}$ lasers (MacKerrow et al. 1996; Schmitt and McVey 1996). The factor $N_{v}$ is given by the equation

$$
N_{v}=1+\frac{2 \cdot B \cdot D_{\text {range }}}{c}
$$

$B$ is the bandwidth covered by the modulation, $c$ is the speed of light, and $D_{\text {range }}$ is the depth of the target along the optical axis within the resolution area at the target of the receiver telescope. The equation for $D_{\text {range }}$ is:

$$
D_{\text {range }}=\frac{1.22 \cdot \lambda \cdot R}{D_{\text {rec }}} \cdot \tan (\theta)
$$


where $\lambda$ is the wavelength of light, $R$ is the distance between the receiver telescope and the target, $D_{\text {rec }}$ is the diameter of the receiver telescope, and $\theta$ is the angle between the optical axis and the normal to the target. For example, with a wavelength of $10 \mu \mathrm{m}$, a telescope diameter of $10 \mathrm{~cm}$, a range of $10 \mathrm{~km}$, angle of $45^{\circ}$, and a bandwidth of $10 \mathrm{GHz}$ gives $N_{v}$ of 82 . This predicts a factor of 9 improvement in SNR for a given measurement time or a factor of 82 decrease in measurement time for a given SNR. Verification of this reduction in speckle noise by FM is a major goal of this task.

The primary goal for FY01 was to build a laboratory system with which to begin testing the performance of FM-LIDAR and the prediction of improved speckle averaging. Following positive results from the laboratory experiments, our ultimate goal is developing a fieldable system to characterize realworld performance and provide verification of a more detailed numerical model of FM-LIDAR.

\subsection{The FM Lidar System and Key Results}

This year significant progress was realized in building a FM-LIDAR system. This system was developed entirely in FY01, and is now operating and used for laboratory tests at ranges of up to $15 \mathrm{~m}$ (50 feet). The general setup for initial experiments is shown in Figure 4. This FM-LIDAR system has several key subsystems that correspond to blocks in our numerical model of FM-LIDAR. Each

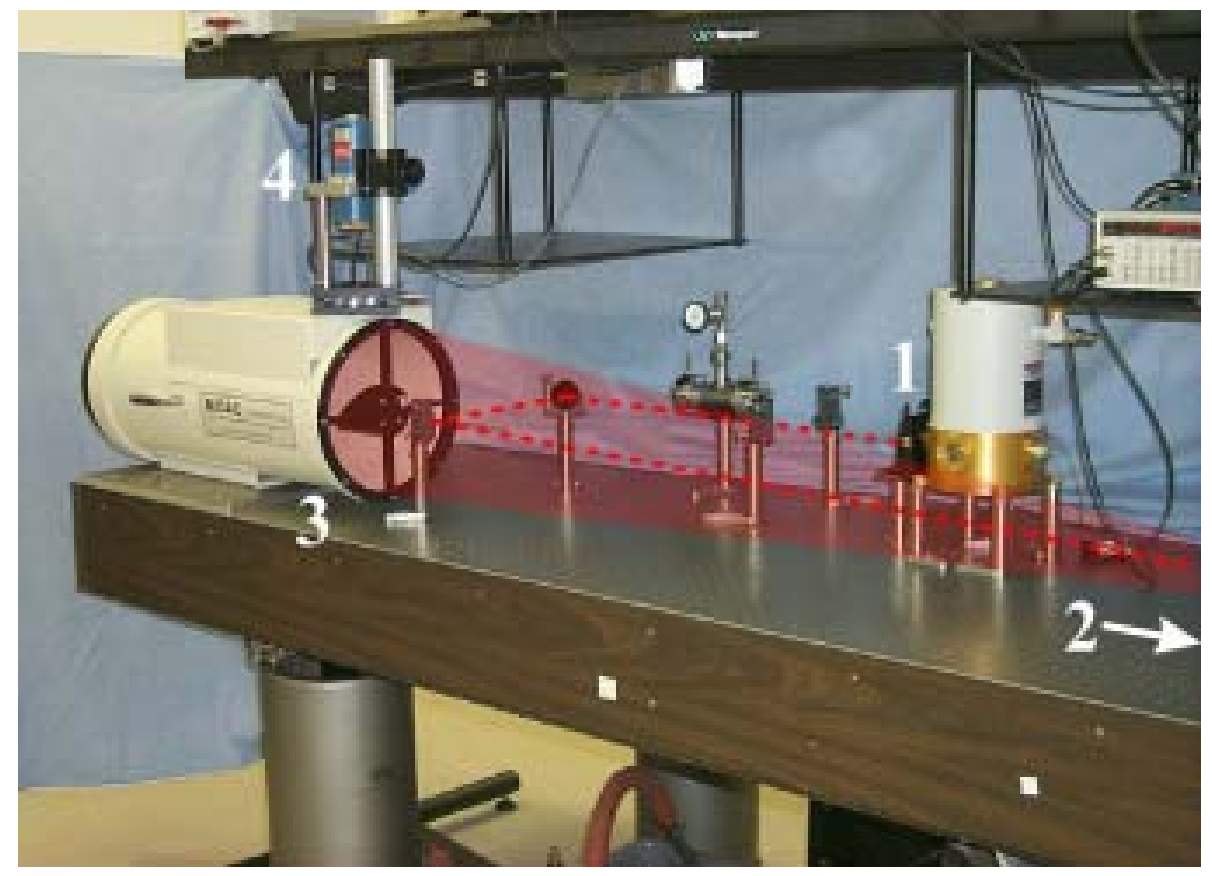

Figure 4. The Source (1) is a Quantum Cascade Laser (QCL) Operating at a Wavelength of $8.2 \mu \mathrm{m}$ and Capable of Delivering up to $30 \mathrm{~mW}$ of Continuous-Wave (cw) Power in a Narrow Optical Bandwidth. The output of the laser is collimated using a short focal length lens and directed toward a scattering target (2). The scattered light is then collected by a 10" Midac telescope (3) and imaged onto a Mercury Cadmium Telluride (MCT) photovoltaic detector (4) where the optical signal is transformed into an electrical signal. 
subsystem may be studied separately and should be optimized so that the total system performs optimally. Characterization of individual subsystems also allows verification of isolated blocks in our numerical models. These subsystems are QCL source, collection optics, detector/transducer, and detection electronics. Each of these has been systematically characterized, paying attention to possible optimizations for a fieldable system.

\subsubsection{MCT Detector and Preamplifier}

An important aspect of an FM-LIDAR system is the ability to detect small returned light signals collected by the telescope. This requires that the detector subsystem be optimized for high gain and low noise. With an ideal detector-preamplifier, this noise would be shot noise that results from the statistics of photons arriving at the detector element, and speckle noise from the interference between light scattered from different points on a rough target. The properties of MCT detectors used for long wavelengths make it very difficult to approach this ideal for low light levels in contrast to what can be done in the visible and near-IR. A thorough understanding of the characteristics of the MCT detector, signal and background light levels, required electrical bandwidth, and preamplifier noise sources is needed to fully optimize a MCT detector-preamp combination for a particular task. We characterized the MCT detector that we are using for our laboratory FM-LIDAR experiments along with a low-noise highbandwidth $(100 \mathrm{MHz})$ preamplifier. While this preamplifier works well for the laser locking experiments for which it was developed, we found that it has significant limitations for use in the FM-LIDAR apparatus and identified several changes that need to be made to tailor this preamp for our FM-LIDAR.

Detector signal properties were tested using an extended blackbody radiation source, allowing the entire field of view (FOV) of the detector to be filled with a known uniform source. Detector I-V curves were recorded for a range of blackbody temperatures from $300 \mathrm{~K}$ to $575 \mathrm{~K}$. This data may be used to calculate a variety of properties of the detector-for instance, the dynamic resistance, linearity, and quantum efficiency as functions of bias voltage. Detector-preamplifier noise properties were examined using a spectrum analyzer and a blackbody source. The noise spectrum was recorded from $12 \mathrm{kHz}$ to $100 \mathrm{MHz}$ (Figure 5) and the optimum frequency range is near $1 \mathrm{MHz}$, where the noise is at a minimum of $5 \mathrm{pA} /(\mathrm{Hz})^{1 / 2}$. While the agreement between the observed and simulated data is in good agreement, the small differences suggest that an additional level of refinement be added to the modeling process. For instance, the observed increase in the low frequency noise is not reproduced in the simulation. Even at the current level of sophistication, the simulation predicts a higher frequency response than is observed experimentally. The dampening at high frequencies is likely due to stray capacitance that is present in the actual preamplifier board, but was not included in the model.

To date, commercial analog lock-in amplifiers have been used, which have a maximum frequency range of $\sim 100 \mathrm{kHz}$, preventing us from operating in the lowest noise frequency range. To enable us to detect at higher frequencies, we will switch to using double-balanced mixers. Another source of noise that we will reduce is the room-temperature blackbody radiation reaching the detector. Use of a cold narrow bandpass filter centered on the QCL frequency will cut out most of the blackbody (background) radiation and its shot noise, which is a significant contribution to the total noise from the detector. The 


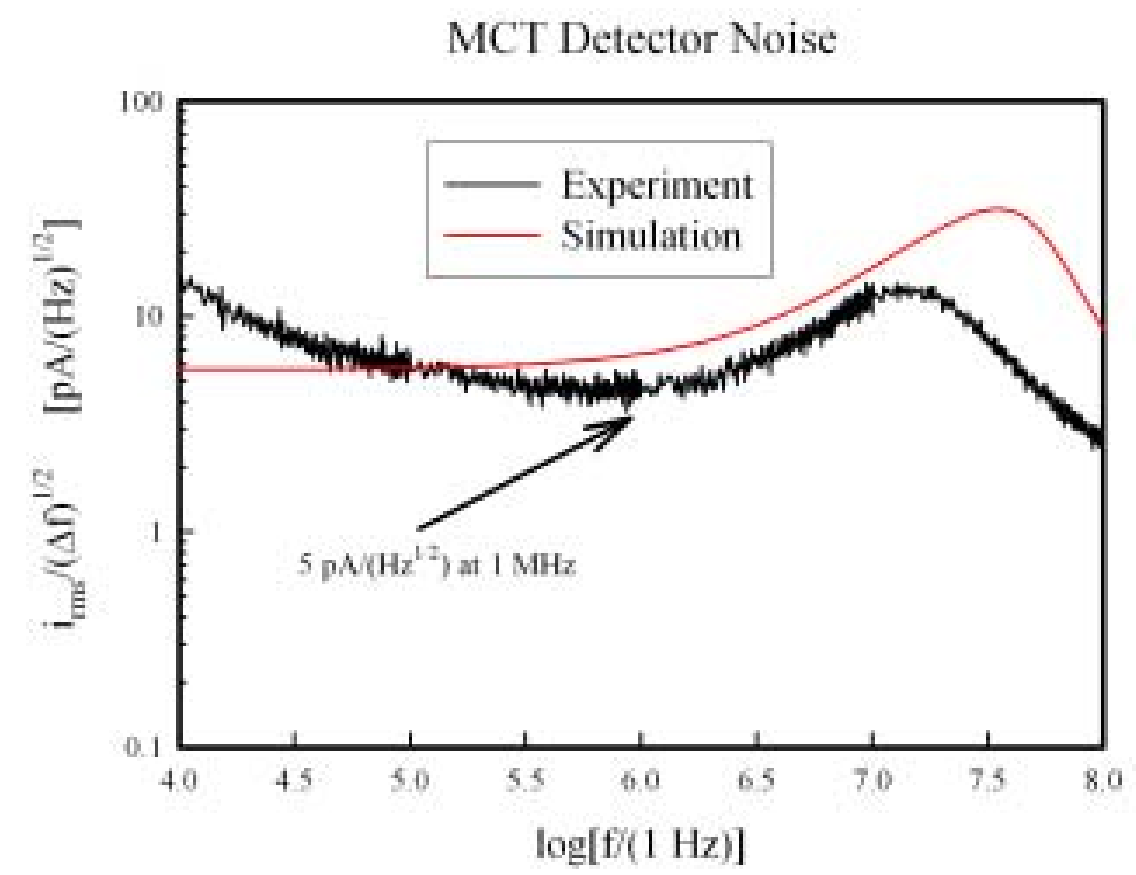

Figure 5. Each Trace was Converted to $\mathrm{V}_{\mathrm{rms}} /(\mathrm{Hz})^{1 / 2}$, and Divided by $5200 \Omega$ (D.C. trans-impedance gain of preamplifier) to get an Input Referred Current Noise

reduced blackbody background may also afford the opportunity to increase the gain of the transimpedance amplifier, which will reduce the noise requirements for the subsequent detection electronics.

\subsubsection{QCL Source}

The QCL that we are using operates near $8.2 \mu \mathrm{m}$ and is tunable over $1.5 \mathrm{~cm}^{-1}$ by varying the current. The output power was measured as a function of current with a threshold at $0.4 \mathrm{~A}, 24 \mathrm{~mW}$ of output at $0.7 \mathrm{~A}$, with the power increasing faster than linearly above $0.6 \mathrm{~A}$. The optical frequency of the QCL was accurately determined using $\mathrm{CH}_{4}$ and $\mathrm{N}_{2} \mathrm{O}$ absorption lines. The match between experiment and the HITRAN database is excellent, indicating a correct frequency calibration. The laser works well between $1226 \mathrm{~cm}^{-1}$ and $1227 \mathrm{~cm}^{-1}$, with the frequency decreasing as the current is increased.

Ideal frequency modulation (FM) of a laser produces no amplitude modulation (AM) until some effect, such as a narrow absorption, converts some of the FM into AM to which a photo-detector will respond. FM of a QCL is most easily accomplished by modulating the current through the QCL, which gives strongly non-ideal FM because there is a large AM that also results from the current modulation. This unwanted AM is frequently called residual amplitude modulation (RAM). This RAM causes a substantial background to the FM signal at the modulation frequency, but if the system has low harmonic distortion, low background measurements can be made at harmonics of the modulation frequency.

Several experiments were done to characterize the harmonic distortion of the QCL/MCT system. A simple LC circuit was used to add modulation to the QCL drive current. Less than $0.4 \%$ harmonic 
distortion was observed $(-47 \mathrm{dBc}$ at $2 f)$ when modulating the current with a sin wave with amplitude large enough to optimally detect a $0.1 \mathrm{~cm}^{-1}$ atmospherically broadened line, and detecting the received signal on the MCT detector. These results indicate that the QCL/detector combination may be operated in a regime where there is low signal distortion.

An important aspect of all FM experiments including FM-LIDAR experiments is to understand the FM and AM characteristics of the laser source, and this is especially true for current-modulated QCLs. Detailed measurements of FM and AM as functions of modulation frequency $(f)$ and QCL current $(i)$ are needed for understanding QCL modulation properties and how to optimize signal detection. The phase difference between the AM and FM is especially interesting because in some cases it can allow one to cancel out the AM background and still retain most of the FM signal. The AM was found to depend significantly on the QCL drive current, as would be predicted from the nonlinearity observed in the power dependence, but its phase is almost independent of drive current. The frequency dependence of the fractional AM is relatively constant for a given current, but the phase lags significantly with increasing frequency. The FM of our QCL shows fairly flat frequency and current dependence; however, the phase lags with increasing frequency. The data plotted in Figure 6 is representative of all observed data. At high frequencies $(100 \mathrm{kHz})$ there is a 45 -degree phase difference, suggesting that with phase-sensitive detection and the proper choice of phase that the RAM background can be cancelled while still retaining $70 \%$ of the FM signal.

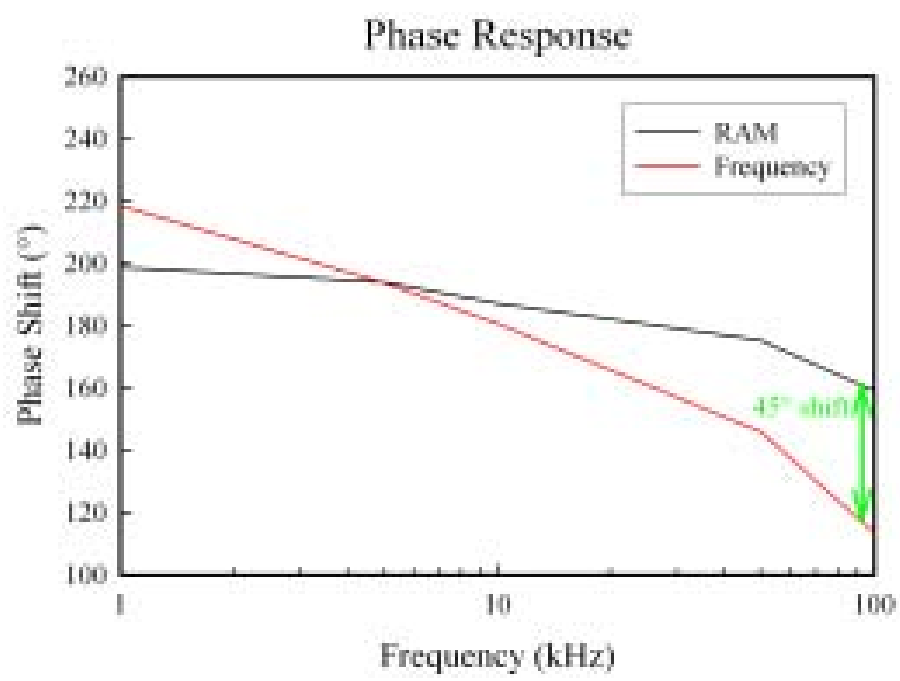

Figure 6. Phase Shift for the Residual Amplitude Modulation and Frequency Modulation

\subsection{Initial Observations of Laser Speckle}

Using a micro-bolometer IR camera, images of the laser beam scattering off single and dual foam targets, which appear to be nearly Lambertian scatterers near $8 \mu \mathrm{m}$, were observed at ranges from 3 to 7 meters (10'-23') from the telescope. Later, experiments were extended to nearly 15 meters (50'), limited by two passes through the lab. Scattering from dual foam targets allows investigating effects due to range depth and monitoring speckle behavior in well-controlled experiments (Figure 7). Scattering 


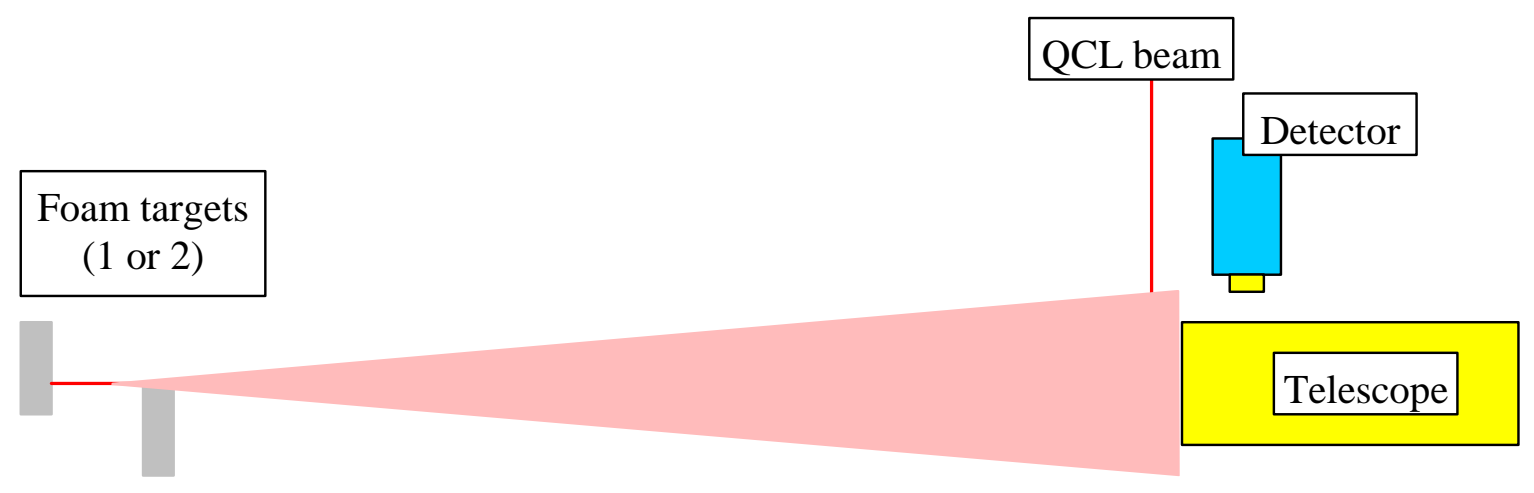

Figure 7. Diagram for Studying Laser Speckle

from single foam targets at various illumination angles mimics situations that may occur when using the FM-LIDAR system from elevated (aircraft) positions. Figure 8 shows a preliminary image of a scattered laser-beam exhibiting speckle.

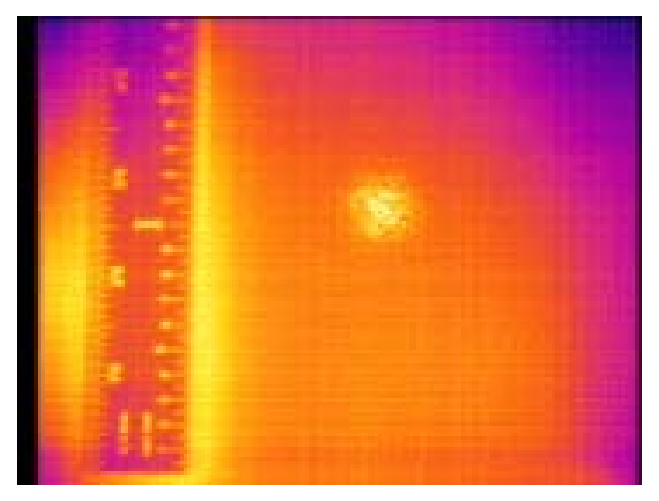

Figure 8. Single Foam Target Speckle Pattern Taken with an IR Bolometer Camera

The numerical model of speckle in FM-LIDAR developed by PNNL predicts several attractive features of FM-LIDAR in mitigating the effects of speckle. Some of these predictions have been verified. Experiments with a single foam target normal to the laser beam produce speckle patterns that do not change with optical frequency. An angled foam target does produce some frequency-dependent speckle; however, it is difficult to get the range depth necessary for measurements in the laboratory. Dual targets were used with the laser clipping the first target (Figure 7). This provides a rigorous test of the equation $\Delta v_{\mathrm{sp}}=\mathrm{c} /\left(2 * D_{\text {range }}\right)$. The data in Figure 9 was collected for $D_{\text {range }}=4 \mathrm{~cm}$, with approximately a $0.4 \mathrm{~cm}^{-1}$ modulation. According to the equation, there should be 3.2 oscillations, which is close to the observed. Further verification of this equation may be done as a function of $\mathrm{D}_{\text {range }}$ and for tilted targets. 


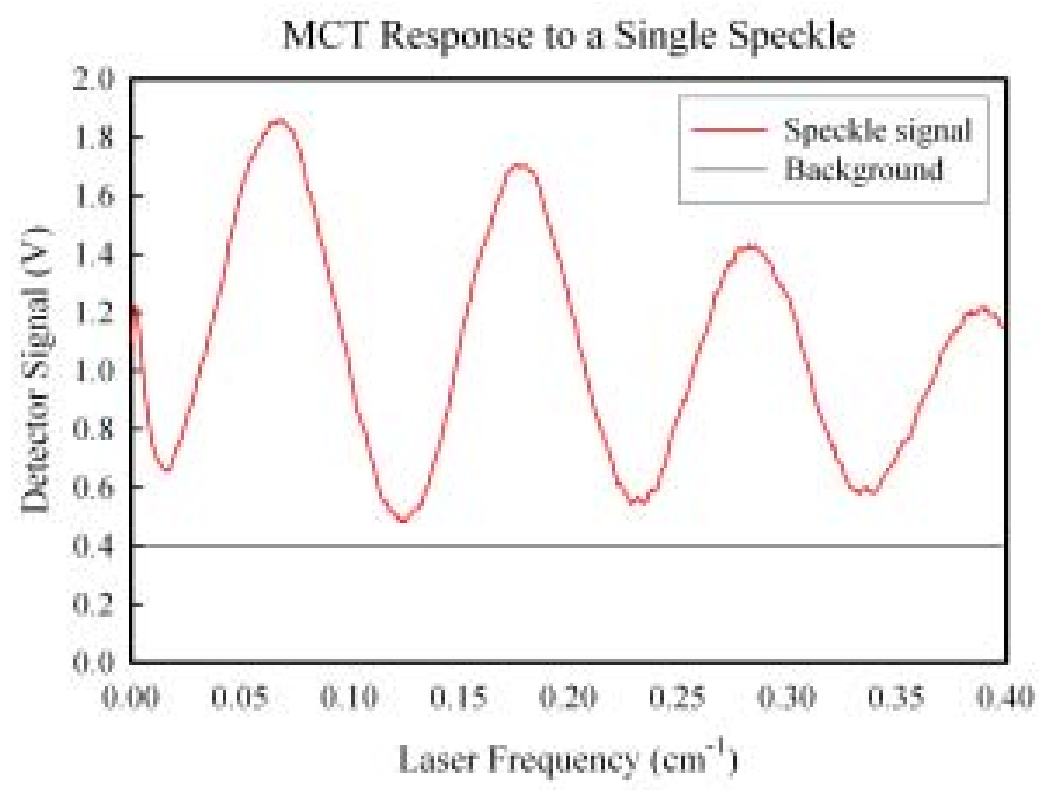

Figure 9. Frequency-Dependent Cycling of Laser Speckle

\subsection{Plans for FY02}

The laboratory FM-LIDAR system was assembled and numerous characterization tests were performed on the working system. The primary thrust for the beginning of FY02 is to provide experimental verification of our model. A twofold approach will be used initially, through geometrical experiments as well as a statistical optics approach. The geometrical approach will allow verification of signal-to-noise formulae presented in the theoretical results through the use of well-controlled apertures, viewing angles, target angles, and range-depth. These are the same parameters that are included in the theoretical predictions for SNR formulae. Showing that the SNR scales in agreement with the predictions will give strong support and validate the theoretical results. The second approach will involve statistical optics concepts where additional properties, such as speckle correlation distance, are examined. This will involve using an infrared camera looking at the image plane formed by the telescope. Of particular interest is examining laser speckle and how quickly it spatially decorrelates with changes in optical frequency. This may be readily determined using two-dimensional autocorrelation procedures on images collected with scattered laser light filling the field of view of the instrument. This would be a direct comparison with the description of speckle given with statistical optics. This spatial decorrelation with frequency is related to how many independent realizations of speckle are included in signal averaging in FM systems. The data from FY01 has given insight on how to modify and enhance the FM-LIDAR system. Improvements will be made on eliminating extraneous blackbody radiation from reaching the detector and extending the capability to modulate at frequencies up to $1 \mathrm{MHz}$ where the noise performance for the detector is optimal. Modulation frequencies $>100 \mathrm{kHz}$ may allow selection of a particular frequency where the FM and AM components are orthogonal ( $90^{\circ}$ out of phase). 


\subsection{Hybrid QC - $\mathrm{CO}_{2}$ Laser Experiments}

\subsection{Introduction}

Long-range experiments for validation of FM-DIAL are not accessible using the optical powers currently achieved with QC lasers. As an interim solution, we propose to injection lock a high-power $\mathrm{CO}_{2}$ laser with a single-mode, narrow line-width QC laser in order to attain the high powers needed for long-range FM DIAL. By using a frequency-modulated QC laser as the master and a high power $\mathrm{CO}_{2}$ laser as the slave, we will be able to reproduce the frequency and phase characteristics of the QC and provide the higher powers needed for field experiments at longer distances.

\subsection{Experiments and Results to Date}

During FY01, two DFB-QC lasers were purchased from the ALPES laser company for injection seeding a $\mathrm{CO}_{2}$ laser and were received in May. Although these devices were not optimal because their wavelengths only matched low-gain transitions, they were the only devices readily available. We expect to receive QC lasers from Lucent for FY02 that have wavelengths matched to the high gain $\mathrm{CO}_{2}$ transitions. After mounting the lasers into a cryogenic Dewar, we set up a custom current controller and a temperature controller to tune the lasers over different $\mathrm{CO}_{2}$ spectral lines. One of the $\mathrm{QC}$ lasers can access two $\mathrm{CO}_{2}$ transitions in the $00^{0} 1-02^{0} 0$ band. At liquid nitrogen temperatures, the $\mathrm{QC}$ accesses the 9R32 line at $1085.765 \mathrm{~cm}^{-1}$. By heating the QC to $115 \mathrm{~K}$, the QC overlaps the 9R30 line at $1084.635 \mathrm{~cm}^{-1}$. This line is useful because it overlaps a strong ammonia absorption feature, which would allow us to detect ammonia for the field experiments. The wavelength range of the second QC laser matches higher rotational lines of the most abundant isotope, ${ }^{12} \mathrm{C}^{16} \mathrm{O}_{2}$, which do not usually lase. This QC laser could be useful with an isotopic gas mixture of ${ }^{12} \mathrm{C}^{18} \mathrm{O}_{2}$.

Initial experiments involved single pass amplification of the cw-QC laser through the ${ }^{12} \mathrm{C}^{16} \mathrm{O}_{2}$ gain medium. The active laser medium was a sealed low-pressure $\mathrm{CO}_{2}$ laser gas mixture with an active length of $80 \mathrm{~cm}$ and an inner bore diameter of $8 \mathrm{~mm}$. A half-wave plate is used to adjust the polarization of the QC laser, and approximately $7 \mathrm{~mW}$ of the free-running QC light is injected along the optical axis of the resonator. When the QC is tuned to the $9 \mathrm{R} 30$ line and $6 \mathrm{~mA}$ of current is applied to the plasma, $10 \mathrm{~mW}$ of power is achieved. Figure 10 shows the amplified power output for various injected QC powers. These results show that we achieved approximately $35 \%$ amplification with a single pass.

Following these successful experiments, we set up preliminary experiments for the injection-locking scheme. We decided to use a ring configuration to reduce optical feedback onto the QC laser because the injected signal should drive the oscillation in only one direction. The experimental arrangement for the laser system is shown in Figure 11. The cavity layout consists of the low-pressure $\mathrm{CO}_{2}$ discharge tube used in the previous experiments and three mirrors that form a ring cavity. The total length of the ring is $376 \mathrm{~cm}$. The output coupler has a 6-m radius of curvature and a maximum reflectivity at $8.5 \mu \mathrm{m}$, which shifted the gain peak of this laser away from the typical maximum of $10.6 \mu \mathrm{m}$ and towards the $9.6 \mu \mathrm{m}$ band. In the ring configuration, self-oscillation occurs predominantly on the 9P20 line at $1046.854 \mathrm{~cm}^{-1}$, 


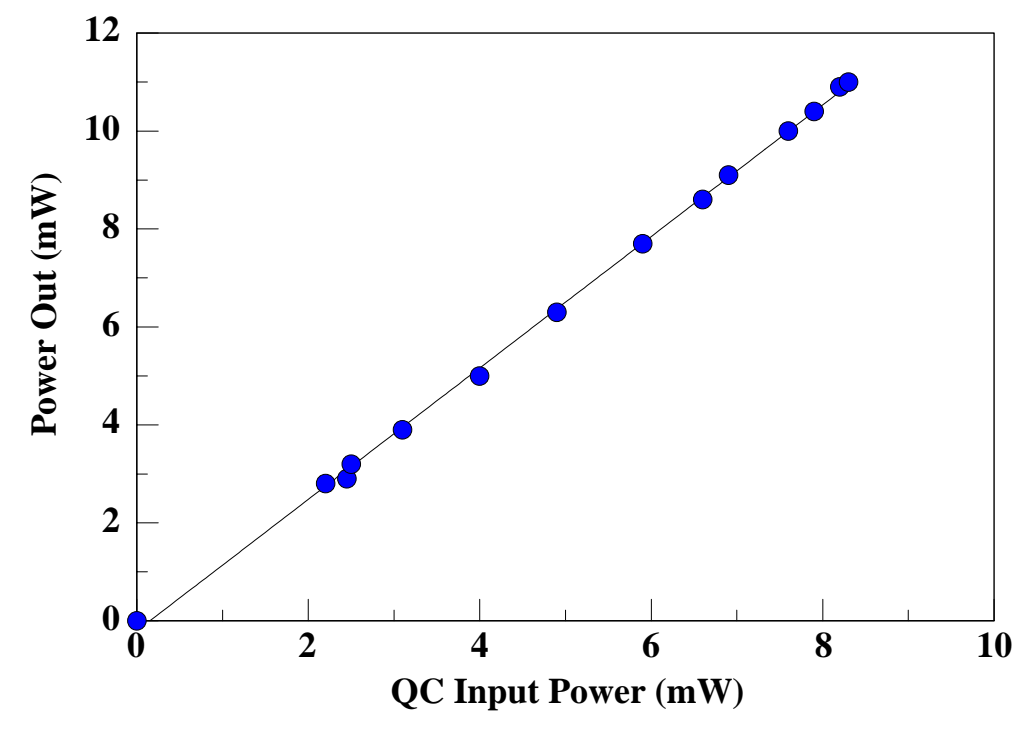

Figure 10. Amplified Power Output vs. QC Power Input for a Single-Pass $\mathrm{CO}_{2} A m p l i f i e r$
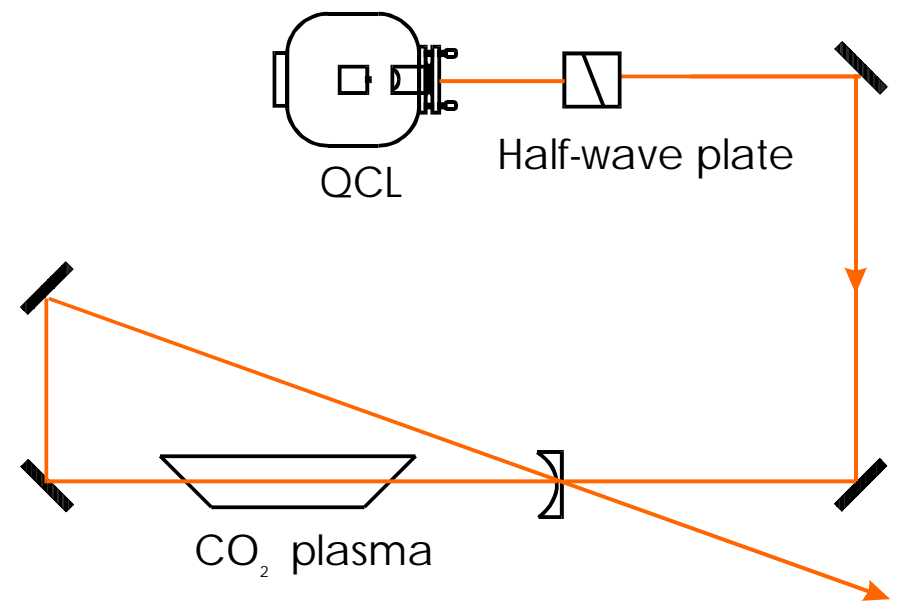

Figure 11. Experimental Set-Up for Injection Locking

but lasing action is also observed on adjacent lines. Furthermore, lasing is observed in the $\mathrm{R}$ band due to mode competition (predominantly on 9R20 at $1078.591 \mathrm{~cm}^{-1}$ ). The laser wavelength is measured using either a $\mathrm{CO}_{2}$ spectrum analyzer or a BOMEM FTIR.

For the $\mathrm{CO}_{2}$ slave laser to be injection-locked by the light coupled into its cavity from the QC master laser, the frequency of the injected light must be within the locking range of the oscillator. This locking range depends on the ratio of the injected input power to the free-running output power, the round-trip transit time, and the cavity losses. For injected frequencies that are outside of this locking range, the output from the slave laser could consist of both the amplified master and self-oscillation frequencies, could have its lasing frequency "pulled" towards that of the master, or could have no measurable effect if 
the injected power is too far below threshold. In other words, for a given injected power, there is a range of frequencies for which the slave laser's frequency will lock to the master laser's frequency.

With the QC beam aligned through the output coupler, only $0.5 \mathrm{~mW}$ of power is injected into the ring cavity because most of the QC power is reflected from the high reflectivity output coupler. Figure 12 shows the FTIR spectrum from the superposed output from the master and slave laser fed into the FTIR. There is no evidence for injection locking, from which we conclude the master frequency is outside of the locking range for the $\mathrm{CO}_{2}$ oscillator. The range available for locking depends on the power of the injected signal, and relatively strong signals are required to capture oscillation on weak or low-gain transitions. In fact, preliminary calculations show the locking range is $<1 \mathrm{MHz}$. To increase the injection locking range, we could decrease the round-trip transit time by reducing the cavity length, increase the coupling of the injected light into the cavity, or decrease the reflectivity of the output coupler. We could also reduce the frequency difference between the master and the slave to within the locking range by incorporating a grating into the cavity to change the slave frequency. Another option is to use a different QC laser that accesses the free-running transitions of the slave.

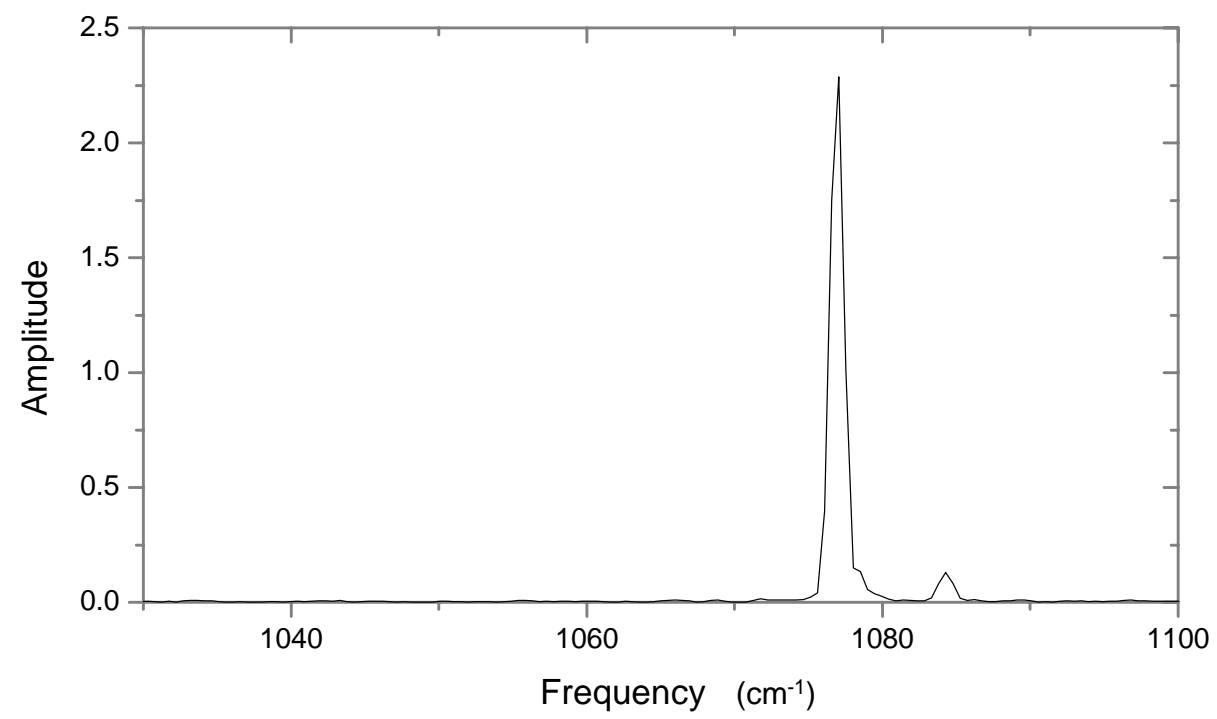

Figure 12. FTIR Spectrum for the Superposed Output of the $\mathrm{QC}$ and the $\mathrm{CO}_{2}$ Laser. The large peak is the self-oscillation of the $\mathrm{CO}_{2}$ laser while the smaller peak is the QC laser oscillation.

\subsection{Future Plans}

In the first quarter of FY02, we will continue analyzing the results and calculating the locking range to determine the requirements for more optimal QC lasers, cavity optics, and cavity configurations. We also plan to mode-match the QC into the ring cavity to increase the coupling of the injected light into the cavity. In order to influence the self-oscillation, it may become necessary to incorporate a grating into the cavity. 
Currently, we are limited to discrete spectral lines in the $\mathrm{CO}_{2}$ laser because we are using a lowpressure $\mathrm{CO}_{2}$ laser. In order to increase the gain width, we plan to incorporate a high-pressure $\mathrm{CO}_{2}$ laser, which could offer almost complete tunability between 9-11 $\mu \mathrm{m}$ due to pressure broadening of the rotational lines. After making the required purchases, we will carry out more detailed experiments for the injection locking and begin using a frequency-modulated QC laser. The resulting laser system will then be used in longer-range FM DIAL experiments.

\subsection{References}

MacKerrow, E. P., J. J. Tiee, M. J. Schmitt, M. C. Whitehead, C. R. Quick, and C. B. Fite. 1996. "Measurements of Temporal Autocorrelations Effects on $\mathrm{CO}_{2}$ DIAL Singlan Averaging Statistics." CALIOPE ITR Proceedings, p. 371-393.

Schmitt, M., and B. McVey. 1996. "Enhancements to the SONDIAL Lidar Design Code." CALIOPE ITR Proceedings, p. 361-370.

Sheen, D. M. 2000. Frequency Modulation Spectroscopy Modeling for Remote Chemical Detection. PNNL-13324, Pacific Northwest National Laboratory, Richland, Washington. 